Moreno-Murcia, J.A. \& Silveira, Y. (2015). Perfiles motivacionales de estudiantes universitarios. Procesos de estudio y satisfacción con la vida. Revista Electrónica Interuniversitaria de Formación del Profesorado, 18 (3), 169-181.

\title{
Perfiles motivacionales de estudiantes universitarios. Procesos de estudio y satisfacción con la vida
}

\author{
Juan Antonio Moreno-Murcia, Yolanda Silveira Torregrosa \\ Universidad Miguel Hernández de Elche
}

\section{Resumen}

El objetivo de este trabajo ha sido determinar los diferentes perfiles motivacionales en estudiantes universitarios y ver su relación con los procesos de estudio (superficial y profundo) así como con la satisfacción que tiene el estudiante con su vida en general. La muestra estuvo compuesta por 431 estudiantes universitarios (202 hombres y 220 mujeres) con una edad media de 22 años (DT = 2.41). Los instrumentos utilizados fueron la Escala de Motivación Académica, Cuestionario Revisado de Procesos de Estudio y la Escala de Satisfacción para la Vida. Se calcularon los estadísticos descriptivos (medias y desviaciones típicas), la consistencia interna y se realizó un análisis clúster y de varianza univariados. Los resultados muestran dos perfiles motivacionales, un perfil más autodeterminado con puntuaciones bajas en desmotivación y altas principalmente en motivación intrínseca de conocimiento, de logro y regulación identificada y un perfil menos autodeterminado con puntuaciones altas en desmotivación y moderadas en el resto de variables motivacionales. Además, los estudiantes pertenecientes al perfil más autodeterminado presentaron menor puntuación en los procesos de estudio superficial, una mayor puntuación en el proceso de estudio profundo estando más satisfechos con la vida a diferencia de los estudiantes del perfil menos autodeterminado.

\section{Palabras clave}

Perfiles motivacionales; motivación académica; procesos de estudio; satisfacción con la vida.

\section{Contacto:}

Juan Antonio Moreno Murcia. Correo electrónico: j.moreno@umh.es, Universidad Miguel Hernández de Elche. Departamento de Psicología de la Salud. Avenida de la Universidad, s/n. 03202 Elche (Alicante). Tel: +34 965222441 


\title{
Motivational profiles of college students. Study process and satisfaction with life
}

\begin{abstract}
The aim of this study is to determine the different motivational profiles in college students and their relationship to study processes (superficial and deep) and the satisfaction with life in general. The sample included 431 college students (202 men and 220 women), with a mean age of 22 years $(S D=2.41)$. The instruments used were: the Academic Motivation Scale, a revised version of the Study Process Questionnaire and the Satisfaction with Life Scale. Descriptive Analyses were performed (mean and standard deviation), bivariate correlation and cluster and univariate analysis. The results indicated two motivational profiles, a more self-determined profile with low amotivation and high scores on knowledge and achievement intrinsic motivation, and identified regulation and a profile less self-determined with high scores on amotivation and moderate motivational in other variables. In addition, students in a self-determined group had lower surface approach, higher deep approach and higher life's satisfaction than students in less self-determined profile.
\end{abstract}

\section{Key words}

Motivational profiles; academic motivation; study process; life's satisfaction

\section{Introducción}

La Cada vez nos encontramos mayor interés en analizar cómo aprenden los estudiantes en la universidad, especialmente, tras la adaptación de las titulaciones al modelo propuesto por el Espacio Europeo de Educación Superior (EEES). Las universidades se han visto involucradas en un proceso de restructuración, las cuales ponen el énfasis en favorecer y potenciar el desarrollo de capacidades y competencias que permitan al estudiante desenvolverse y adaptarse a las demandas de una sociedad en continuo cambio. El estudio de los procesos implicados en el aprendizaje se presenta como elemento influyente en el proceso educativo dada su relación con éxito y rendimiento académico (Bernardo, 2003; Mpofu y Oakland, 2001; Muñoz y Gómez, 2005). Son muchos los factores involucrados en el proceso de aprendizaje de los estudiantes, siendo en este estudio los aspectos cognitivos y motivacionales el elemento central de este trabajo. Conocer cómo se relacionan las diferentes variables motivacionales y cognitivas permitirá al docente poner en marcha las estrategias necesarias para lograr un mejor rendimiento en el alumnado.

El entorno de aprendizaje creado por el docente determinará la implicación, la manera de aprender y el modo en el que los estudiantes se enfrentan a las tareas de aprendizaje. Es decir, la metodología empleada por el docente y la forma en que evalúa a sus estudiantes puede influir significativamente en la forma de aprender de los mismos (Gargallo, 2006, 2008 y 2009). De ahí la importancia del papel del docente, el cual debe ofrecer una enseñanza centrada en el estudiante, dándole un mayor protagonismo y autonomía empleando metodologías activas y participativas para lograr un mayor compromiso en el alumnado y conseguir que sean ellos mismos los que construyan su propio conocimiento y adquieran las diferentes competencias. Para ello es fundamental generar interés en el 
alumnado y motivarle a "aprender a aprender" y se implique así de forma activa en el proceso educativo.

Existe cierta relación entre la motivación y el enfoque de aprendizaje adoptado, de manera que el tipo de motivación predominante está relacionado con las diferentes estrategias de aprendizaje que utiliza el estudiante para alcanzar sus objetivos (Pozo, Monereo y Castelló, 2005) así como con el enfoque de aprendizaje adoptado por el estudiante (Biggs, 1989, Entwistle, 1987). La relación existente entre motivo-estrategia, es a lo que se denomina Enfoque de Aprendizaje (Biggs, 1985), y es la que lleva al estudiante ser consciente de sus motivos y a elegir la estrategia más adecuada en su tarea. Son principalmente dos los enfoques de aprendizaje, enfoque profundo y superficial (Biggs, Kember, y Leung, 2001; Entwistle, 1985; Hernández Pina, 2000; González Geraldo, del Rincón Igea, y Delio, 2011) los cuales están compuestos de elementos situacionales y personales (Biggs, 1993). Los estudiantes que adoptan principalmente un enfoque profundo se caracterizan por presentar un rol activo, presentan una mayor motivación intrínseca y adoptan estrategias que le llevan a satisfacer su interés por comprender lo que aprende, tratando de relacionarlo con sus conocimientos previos (Doménech, y Gómez, 2011). Los estudiantes con enfoque superficial se caracterizan por presentar una mayor motivación extrínseca y por utilizar estrategias basadas en el aprendizaje memorístico, reproduciendo de forma mecánica lo que aprende (Barca, 2005) y por tener un mayor temor al fracaso (Biggs, Kember, y Leung, 2001), conformándose con cumplir con los objetivos (aprobar un examen, pasar de curso, etc.). Las estrategias utilizadas para enfrentarse a las tareas propuestas por el docente en el proceso educativo condicionan la forma en la que se aprende, de manera que el estudiante adquiere e interioriza las estrategias y en función de la situación elegirá cómo y dónde aplicarlas dada su transferencia y naturaleza flexible (Monereo, 2000).

Las personas, según la Teoría de la Autodeterminación (TAD) de Deci y Ryan (1991, 2000), tienden a comportarse o aprender en función de sus orientaciones motivacionales. Esta teoría establece los distintos niveles de motivación en un continuo, que va desde la desmotivación hasta la motivación intrínseca (Deci y Ryan, 2000; Reeve et al., 2004, 2008; Ryan y Deci, 2000, 2002). En este continuo se distingue entre motivación autónoma y controlada (Deci y Ryan, 2012) estando la motivación autónoma formada por la motivación intrínseca (de logro, conocimiento y de estimulación) y la regulación identificada, mientras que la regulación externa y la introyectada dan lugar a la motivación controlada (Katz, Assor, y Kanat, 2008; Miquelon y Vallerand, 2006; Miquelon, Vallerand, Grouzet, y Cardinal, 2005; Ratelle, Guay, Vallerand, Larose, y Senécal, 2007). Dado que los estudiantes no solo presentan un tipo de motivación ni utilizan siempre un único enfoque de aprendizaje, pudiendo coexistir motivaciones intrínsecas y extrínsecas para que el estudiante realice una determinada tarea (Harter, 1981; Gillet et al., 2009; Lepper et al., 2005; Ryan et al., 1995) parece interesante llevar a cabo análisis de conglomerados para identificar los diferentes perfiles motivacionales en una misma clase, ya que agrupar a los estudiantes en función de su perfil motivacional permite intervenir de forma más individualizada según las necesidades del grupo (Roeser et al., 1998).

Esta teoría presenta un concepto multidimensional de la motivación y se distingue entre la cantidad o intensidad de la motivación y la calidad de la misma. De manera que no solo nos va a interesar que los estudiantes tengan un nivel elevado de motivación sino que este sea de buena calidad (Vansteenkiste, Soenens, Sierens, Luyckx, y Lens, 2009). Encontramos diversos trabajos que han estudiado cómo se combinan los diferentes tipos de motivación (Kusurkar et al., 2013; Lin et al., 2003; Ratelle et al., 2007; Vansteenkiste et al., 2009; Wormington, Corpus, y Anderson, 2011). En la mayoría de ellos se han identificado cuatro perfiles motivacionales. Un perfil con altas puntuaciones en motivación intrínseca y 
extrínseca (alta cantidad), un perfil con puntuaciones altas en motivación intrínseca y bajas en motivación extrínseca (buena calidad), un perfil con puntuaciones bajas en motivación intrínseca y altas en motivación extrínseca (pobre calidad) y un perfil con puntuaciones bajas en ambas (baja cantidad). Los estudiantes con un perfil con puntuaciones altas en motivación intrínseca y bajas en motivación extrínseca presentan mayor persistencia y compromiso con las tareas escolares (Deci y Ryan 2002; Vansteenkiste et al., 2009). Aunque encontramos trabajos (Lepper et al., 2005; Lin et al., 2003; Ratelle et al., 2007; Rodríguez Ayán, 2010) que muestran como un perfil con altas puntuaciones en ambas motivaciones podría ser adaptativo.

Además la teoría de la autodeterminación, determina que hay tres necesidades psicológicas básicas (percepción de competencia, autonomía y relación con los demás) que deben satisfacerse a fin de promover el bienestar psicológico y un desarrollo saludable (Deci y Ryan, 2000). Los procesos de aprendizaje autónomo se asocian positivamente con un mayor bienestar psicológico, autoestima, autoconcepto académico. Siendo la motivación intrínseca un predictor del bienestar psicológico independientemente del rendimiento (Chirkov y Ryan, 2001; Burton, Lydon, D’alessandro y Koestner, 2006). Las conductas más controladas se han relacionado con baja autoestima, elevados niveles de ansiedad, bajos niveles de bienestar subjetivo (Ryan y Connell, 1989; Sheldon y Elliot, 1999). En este sentido, la variable satisfacción con la vida se considera un indicador importante del bienestar psicológico positivo (Diener, Emmons, Larsen, y Griffin, 1985; Huebner et al. 2005).

Así pues, el objetivo se centró en identificar los perfiles motivacionales en los estudiantes universitarios y comprobar su relación con los distintos enfoques adoptados a la hora de aprender así como con su posible relación en la satisfacción con la vida de los mismos. Por lo que, se espera que un perfil motivacional más auto-determinado se relacionará de forma positiva con un enfoque de aprendizaje profundo dando lugar a una mayor satisfacción con la vida en los estudiantes universitarios.

\section{Metodología}

\section{Participantes}

La muestra estuvo compuesta por 431 estudiantes universitarios (202 hombres y 220 mujeres) de $1^{\circ}$ a $4^{\circ}$ curso, pertenecientes a los grado de Ciencias de la Salud de la Universidad Miguel Hernández de Elche, con una edad media de 22 años (DT = 2.41).

\section{Medidas}

Motivación Académica. Para medir la motivación académica del estudiante se empleó la versión traducida y validada al español de Núñez, Martí-Albo, y Navarro (2005) de la Échelle de Motivation en Éducation (EME) (Vallerand, Blais, Brière y Pelletier, 1989). El instrumento está formado por 28 ítems, precedidos por la frase “¿Por qué estudias?” y distribuidos en siete subescalas cinco de ellas de cuatro ítems y las dos restantes de tres: desmotivación (e.g. "No sé por qué voy al instituto y, sinceramente, no me importa"), regulación externa (e.g. "Para poder conseguir, posteriormente, un mejor salario"), regulación introyectada (e.g. "Porque cuando hago bien las tareas en clase me siento importante"), regulación identificada (e.g. "Porque me ayudará a tomar una mejor decisión en lo que respecta a mi orientación profesional”), motivación intrínseca al conocimiento (e.g. "Porque mi estudios me permiten seguir aprendiendo muchas cosas que me interesan"), motivación intrínseca al logro (e.g. "Por la satisfacción que siento cuando voy superando actividades académicas difíciles") y motivación intrínseca a las experiencias estimulantes (e.g. "Porque realmente me gusta asistir a clase"). Las respuestas se puntuaron de acuerdo a una escala tipo Likert 
de siete puntos, desde 1 (no se corresponde en absoluto) hasta 7 (se corresponde totalmente). El valor de alfa obtenido en este estudio fue de .84 para desmotivación y para regulación externa, .80 para regulación introyectada y para regulación identificada, .84 para motivación intrínseca al conocimiento, .81 para motivación intrínseca al logro y .74 para motivación intrínseca a las experiencias estimulantes. Para evaluar la motivación autodeterminada se utilizó el índice de autodeterminación (IAD) que se ha mostrado como válido y fiable en diferentes trabajos (Chantal, Robin, Vernat, y Bernache-Asollant, 2005; Kowal y Fortier, 2000) y se calcula con la siguiente fórmula: $(2 \times(\mathrm{MI}$ conocimiento $+\mathrm{MI}$ ejecución + MI estimulación $) / 3)+((M E$ identificada $+M E$ integrada/2 $))-((M E$ externa $+M E$ introyectada)/2) - (2 x Desmotivación) (Vallerand, 1997). Se obtuvo un alfa de Cronbach de .87 .

Procesos de Estudio. Se empleó el Cuestionario Revisado de Procesos de Estudio (R-CPE-2F), de Recio y Cabrero (2005), en su versión en español. Está compuesto por 20 ítems con dos categorías de enfoques de aprendizaje: profundo (DA) y superficial (SA), y un recorrido de 5 opciones, con cuatro subescalas: motivación profunda (DM) (e.g. "En ocasiones el estudio me proporciona un sentimiento de profunda satisfacción personal"), estrategia profunda (DS) (e.g. "Dedico gran parte de mi tiempo libre a recopilar más información sobre temas interesantes ya tratados"), motivación superficial (SM) (e.g. "No encuentro mi curso muy interesante, por eso trabajo lo mínimo") y estrategia superficial (SS) (e.g. "Puedo aprobar la mayoría de los exámenes memorizando partes clave de los temas, y no intentando comprenderlos"). La escala Likert iba desde 1 (nunca o casi nunca es verdadero para mí) hasta 5 (siempre o la mayoría de las veces es verdadero para mí). Los ítems fueron precedidos de la frase "En mi curso...". La consistencia interna obtenida fue de .78 tanto para estudiante profundo como para el superficial.

Satisfacción con la vida. Se utilizó la Escala de Satisfacción para la Vida (ESDV-5) de Vallerand, Blais, Briére, y Pelletier (1989) validada al contexto español (Atienza, Balaguer, y García-Merita, 2003; Atienza, Pons, Balaguer, y García-Merita, 2000). Está formada por cinco ítems agrupados en un solo factor (e.g. "En general, mi vida se corresponde con mis ideales"). La sentencia previa fue "Satisfacción con tu vida...". Las respuestas fueron valoradas a través de una escala tipo Likert que oscilaba desde 1 (Totalmente en desacuerdo) a 7 (Totalmente de acuerdo). La consistencia interna fue de .86.

\section{Procedimiento}

Se contactó con los docentes implicados para informarles del objetivo de la investigación y solicitarles su colaboración para que los estudiantes pudieran rellenar los cuestionarios en su tiempo de clase. Los cuestionarios se administraron en su mayoría en las clases prácticas para garantizar un mayor número de estudiantes, dada la obligatoriedad de las mismas. Siempre bajo la supervisión del investigador, que les explicaba sobre cómo cumplimentar los cuestionarios y solventar así posibles dudas que pudieran surgir durante el proceso. Se insistió en el anonimato de las repuestas y en que contestaran con sinceridad. El tiempo requerido para su cumplimentación fue de 15 minutos, aproximadamente.

\section{Análisis de datos}

En primer lugar, se calcularon los estadísticos descriptivos de todas las variables objeto de estudio (medias y desviaciones típicas), se analizó la consistencia interna de cada factor mediante el coeficiente de Alfa de Cronbach. A continuación, se trató de identificar diferentes perfiles motivacionales. Se realizó un análisis jerárquico de clúster con método Ward, utilizando todas las variables motivacionales de la escala de motivación académica. A continuación, con las mismas variables se trató de confirmar la solución de perfiles hallada, utilizando un análisis de conglomerados de K medias. Para examinar las características de 
cada perfil motivacional de acuerdo a las variables de procesos de estudio y satisfacción con la vida, se realizaron análisis de varianza univariados (ANOVA). El registro y posterior tratamiento de los datos se llevó a cabo con el paquete estadístico SPSS 21.0.

\section{Resultados}

\section{Análisis de cluster}

Para la realización del análisis de cluster, se siguieron las fases propuestas por Hair et al. (1998). En primer lugar, se observó la existencia de casos perdidos en algunas de las variables estudiadas, siendo excluidos de la muestra de estudio. En segundo lugar, se estandarizaron todas las variables usando las puntuaciones $Z$, no encontrando ninguna puntuación por encima de 3, lo que implicó la inexistencia de clasificaciones outliers o casos perdidos en la totalidad de la muestra. En el siguiente paso, la distribución univariada de todas las variables agrupadas fue examinada para su normalidad.

Para determinar los grupos motivacionales, se realizó un análisis de conglomerados jerárquicos utilizando el método Ward. El dendograma obtenido sugirió la existencia de dos grupos (Tabla 1). Para decidir la adecuación de los grupos surgidos nos basamos en el incremento de los coeficientes de aglomeración. De acuerdo con Norusis (1992), los coeficientes pequeños indican gran homogeneidad entre los miembros del cluster, mientras que, por el contrario, los coeficientes grandes muestran grandes diferencias entre sus miembros. Tras el análisis se obtuvieron dos perfiles motivacionales (Figura 1): un perfil autodeterminado (cluster 1) con puntuaciones bajas en desmotivación, moderadas en regulación extrínseca y altas principalmente en motivación intrínseca de conocimiento, de logro y regulación identificada, y un perfil menos autodeterminado (cluster 2) con puntuaciones altas en desmotivación, moderadas en regulación extrínseca y bajas en el resto de variables motivacionales, siendo la motivación intrínseca al logro la más baja (Tabla 1).

Tabla 1.

Análisis diferencial y valores Estandarizados, Medias y Desviaciones Típicas de las Variables en cada Cluster

\begin{tabular}{|c|c|c|c|c|c|c|c|c|}
\hline \multirow[t]{2}{*}{ Variables } & \multicolumn{3}{|c|}{$\begin{array}{l}\text { Cluster } 1 \\
(n=277)\end{array}$} & \multicolumn{3}{|c|}{$\begin{array}{l}\text { Cluster } 2 \\
(n=154)\end{array}$} & \multirow[b]{2}{*}{$F$} & \multirow[b]{2}{*}{$p$} \\
\hline & $M$ & DT & Z & $M$ & DT & Z & & \\
\hline 1. Desmotivación & 1.29 & .43 & -.43 & 2.60 & 1.36 & .78 & 217.82 & .00 \\
\hline 2. Regulación externa & 4.76 & 1.43 & .13 & 4.24 & 1.35 & -.24 & 13.80 & .00 \\
\hline 3. Regulación identificada & 6.02 & .86 & .41 & 4.74 & 1.04 & -.74 & 187.25 & .00 \\
\hline 4. Regulación introyectada & 5.01 & 1.15 & .38 & 3.56 & 1.20 & -.68 & 149.96 & .00 \\
\hline 5. MI conocimiento & 5.72 & .84 & .42 & 4.45 & .96 & -.76 & 201.92 & .00 \\
\hline 6. MI al logro & 5.70 & .76 & .50 & 4.15 & .94 & -.90 & 347.66 & .00 \\
\hline 7. MI estimulación & 4.63 & 1.09 & .35 & 3.45 & 1.02 & -.63 & 120.18 & .00 \\
\hline
\end{tabular}

Nota: $\mathrm{MI}=$ motivación intrínseca 


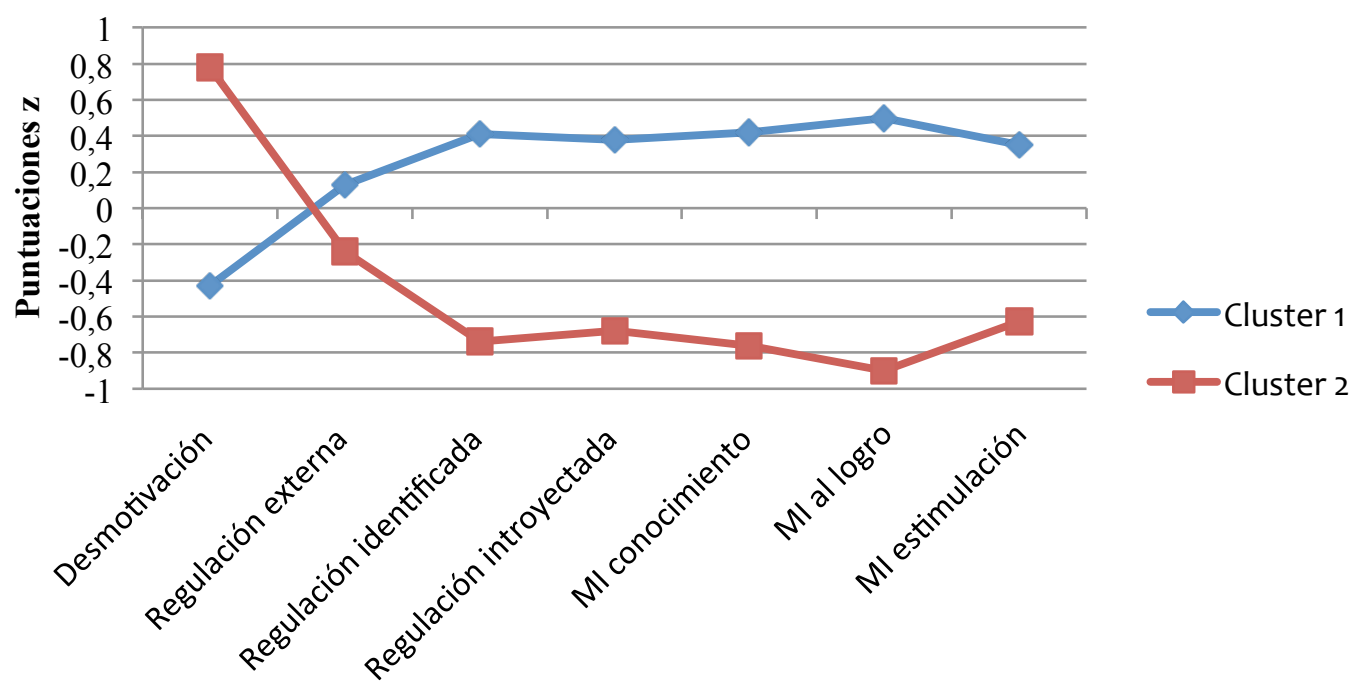

Figura 1. Análisis de Conglomerados Jerárquicos con Método Ward

Análisis diferencial de los tipos de motivación según los procesos de estudio y la satisfacción con la vida

Para examinar las características de cada perfil motivacional de acuerdo a las variables: procesos de estudio (superficial y profundo) y satisfacción con la vida, con la muestra total se realizó un análisis diferencial con los clusters como variables independientes y los procesos de estudio y satisfacción con la vida como variables dependientes. Se encontraron diferencias (Lambda de Wilks $=.83, \mathrm{~F}(3,427)=28.46, \mathrm{p}<.01, \mathrm{\eta} 2=.17)$ entre los diferentes clusters en las tres variables (Tabla 2), mostrando los datos, que los estudiantes con un perfil más autodeterminado presentaban procesos de estudio más profundos y estaban más satisfechos con la vida que aquellos que presentaban un perfil menos autodeterminado.

Tabla 2.

Anova de los tipos de motivación según los procesos de estudio y la satisfacción con la vida

\begin{tabular}{|l|c|c|c|c|c|c|}
\hline & \multicolumn{2}{|c|}{$\begin{array}{l}\text { Cluster 1 } \\
\text { Variables }\end{array}$} & \multicolumn{2}{|c|}{$\begin{array}{l}\text { Cluster 2 } \\
(n=277)\end{array}$} & & \\
\hline & $M$ & DT & $M$ & DT & $F$ & $\eta^{2}$ \\
\hline 1. Procesos Estudio Superficial & 2.22 & .60 & 2.49 & .68 & $18.76^{* *}$ & .04 \\
\hline 2. Procesos Estudio Profundo & 3.12 & .57 & 2.78 & .57 & $35.54^{* *}$ & .08 \\
\hline 3. Satisfacción con la vida & 5.64 & .93 & 4.89 & 1.25 & $50.67^{* *}$ & .11 \\
\hline
\end{tabular}

Nota: $p<.01^{* *}$

\section{Discusión y conclusiones}

Conocer cuáles son los motivos que llevan al estudiante a querer aprender y cómo van a conseguirlo es clave para una intervención docente eficaz, permitiendo al docente diseñar las estrategias más adecuadas para suscitar interés en el alumnado y potenciar el desarrollo 
de habilidades y las competencias necesarias para lograr un aprendizaje más profundo, autónomo y duradero. Por lo que el objetivo de este trabajo se ha centrado en identificar los perfiles motivacionales en un contexto universitario y relacionarlos con los enfoques de enseñanza adoptados y con la satisfacción con la vida. Los resultados obtenidos corroboran la hipótesis planteada ya que los estudiantes pertenecientes al perfil autodeterminado presentaron un mayor uso de procesos de estudios profundo y mayor satisfacción con la vida que los pertenecientes al perfil menos autoderminado.

En este estudio se han identificado dos perfiles motivacionales (uno más autoderminado (mayores puntuaciones de motivación intrínseca y bajas en desmotivación) y otro menos autodeterminado (mayores niveles de desmotivación, moderados de regulación externa y bajos de motivación intrínseca). A pesar de que en otros trabajos se han identificado cuatro perfiles (un perfil con altas ambas motivaciones, un perfil con alta motivación intrínseca y baja extrínseca, un perfil con alta motivación extrínseca y baja intrínseca y un último perfil con bajas ambas motivaciones) como en el realizado por Vansteenkiste et al. (2009) o el realizado por Ratelle et al. (2007) con universitarios, donde los resultados de ambos trabajos mostraron que los estudiantes del perfil más autodeterminado (alta motivación intrínseca y baja extrínseca) presentaban un mayor compromiso, motivación y mejor rendimiento que los de otros grupos.

Por otro lado, encontramos investigaciones que afirman que una alta motivación intrínseca junto una moderada motivación extrínseca podría ser adaptativo. En este caso el estudio de Lin et al. (2003) mostró que los estudiantes con una alta motivación intrínseca y moderada motivación extrínseca presentaron comportamientos más adaptativos y un mejor rendimiento que aquellos que tuvieron una alta motivación intrínseca junto una alta o baja motivación extrínseca.

Los estudiantes con un perfil más autodeterminado han sido los que han presentado mayor valoración de los procesos de estudio profundos estando, además, más satisfechos con su vida. Al igual que en otros trabajos, parece que existe una relación entre el tipo de motivación y el enfoque de aprendizaje adoptado para el desempeño de una determinada tarea. De la Fuente, Pichardo, Justicia, y Berbén (2008) ya indicaron una relación negativa entre el enfoque superficial y la autorregulación del aprendizaje. De forma que aquellos universitarios que estudian más profundamente y autorregulan su aprendizaje se muestran más satisfechos. En esta línea, Hernández, Rodríguez, Ruiz, y Esquivel (2010) nos dicen que los estudiantes profundos presentan un elevado interés intrínseco mostrando mayor implicación en las tareas, lo que les lleva a emplear estrategias profundas, a tener una mayor autorregulación y a estar más satisfechos con el aprendizaje (De la Fuente, et al., 2008).

En relación a la satisfacción con la vida de los estudiantes, los datos muestran que los estudiantes más autodeterminados además de utilizar procesos de estudio profundos están más satisfechos con sus vidas que aquellos que presentan un perfil menos autoderminado. Encontramos investigaciones que muestran que aquellos estudiantes que tienen una actitud positiva hacia el aprendizaje muestran mayor autoeficacia y satisfacción académica influyendo ésta sobre su satisfacción con la vida en general (Lent, 2004; Lent et al., 2005; Lounsbury et al., 2004; Ojeda, Flores, y Navarro, 2011). Por otro lado, según el trabajo de Dishet et al. (2012), la percepción del estudiante sobre el soporte de autonomía aportado por el docente predice la autoeficacia, logros académicos y la satisfacción con la vida de los estudiantes. En la misma línea, Reeve, Jang, Carrell, Barch, y Jeon (2004) nos dice que aquellos que perciben un soporte de autonomía por parte del profesorado están más dispuestos a participar de forma activa en el aprendizaje de las tareas, presentan mayor compromiso y están más satisfechos con su vida. Por lo que el ambiente donde se 
desarrolla el proceso educativo parce cobrar especial importancia, ya que la percepción que tengan los estudiantes puede influir en la motivación y en los resultados de los mismos (Hernández, Rodríguez, Ruiz, y Esquivel, 2010). Promover procesos de autorregulación contribuirá a incrementar la motivación y el aprendizaje académico en los estudiantes (Pintrich, 2004; Rosário, Mourão, Trigo, Núnez y González-Pienda, 2005; Suárez Riveiro, Fernández Suárez y Anaya Nieto, 2005). Por lo que parece, que los enfoques de aprendizaje adoptados por los estudiantes se van perfilando en función de los modelos metodológicos empleados por los docentes (Hernández et al., 2004) siendo la utilización del enfoque profundo el que ofrece una mayor motivación intrínseca, mayor persistencia, compromiso, rendimiento académico (Deci y Ryan 2002; Vansteenkiste et al., 2009), y una mayor satisfacción con la vida.

Alguna de las limitaciones que presenta este trabajo se centra en el análisis de clúster, ya que al ser un estudio correlacional solo se establecen relaciones entre las variables no existiendo relación de causalidad. Sería interesante seguir profundizando en este aspecto, realizando estudios de carácter longitudinal y de intervención, lo que nos aportaría mayor información sobre las relaciones que existen entre los enfoques de aprendizaje adoptados por los estudiantes así como las estrategias utilizadas según los distintos perfiles motivacionales y ver si todo ello puede afectar a su satisfacción con la vida y mejorar el rendimiento académico.

\section{Bibliografía}

Atienza, F. L., Balaguer, I., y García-Merita, M. L. (2003). Satisfaction with Life Scale: analysis of factorial invariance across sexes. Personality and Individual Differences, 35, 12551260.

Atienza, F. L., Pons, D., Balaguer, I., y García-Merita, M. L. (2000). Propiedades psicométricas de la Escala de Satisfacción con la Vida en adolescentes. Psicothema, 12(2), 331-336.

Barca, A. (2005) Atención á diversidade na educación secundaria de Galicia: perfís do alumnado con baixo rendemento escolar e propostas de intervención psicoeducativa. Revista Galega do Ensino, 45, 353-386.

Bernardo, A. B. (2003). Approaches to learning and academic achievement of Filipino students. The Journal of Genetic Psychology, 164, 101-114.

Biggs, J. B. (1985). The role of metalearning in study processes. British Journal of Educational Psychology, 55, 185-212. doi: 10.1111/j.2044-8279.1985.tb02625.x

Biggs, J. B. (1989). Approaches to the enhancement of tertiary teaching. Higher Education Research and Development, 8(1), 7-25.

Biggs, J. (1993). What do inventories of students' learning processes really measure? A theoretical review and clarification. British Journal of Educational Psychology, 63, 319.

Biggs, J., Kember, D., Leung, D. (2001): “The revised two-factor Study Process. Questionnaire: R-SPQ-2F. British Journal of Educational Psychology, 71, 133-149.

Burton, K., Lydon, J., D'Alessandro, D., y Koestner, R. (2006). The differential effects of intrinsic and identified motivation on well-being and performance: Prospective, experimental, and implicit approaches to self-determination theory. Journal of Personality and Social Psychology, 91, 750-762. 
Chantal, Y., Robin, P., Vernat, J. P., y Bernache-Asollant, I. (2005). Motivation, sportspersonship, and athletic aggression: a mediational analysis. Psychology of Sport and Exercise, 6, 233-249.

Chirkov, V., y Ryan, R. M. (2001). Parent and teacher autonomy support in Russian and U.S. adolescents: Common effects on well-being and academic motivation. Journal of Cross-Cultural Psychology, 32(5), 618-635.

Deci, E. L., y Ryan, R. M. (1991). A motivational approach to self: Integration in personality. En R. Dienstbier (Ed.), Nebraska symposium on motivation: Vol. 38. Perspectives on motivation (pp. 237-288). Lincoln, NE: University of Nebraska Press.

Deci, E. L., y Ryan, R. M. (2000). The "what" and "why" of goal pursuits: Human needs and the self-determination of behaviour. Psychological Inquiry, 11, 227-268.

Deci, E. L., y Ryan, R. M. (Eds.) (2002). Handbook of selfdetermination research. Rochester, NY: University of Rochester Press.

Deci, E. L., y Ryan, R. M. (2012). Self-determination theory. En P. A. M. Van Lange, A. W. Kruglanski, y E. T. Higgins (Eds.), Handbook of theories of social psychology: Vol. 1 (pp. 416-437). Thousand Oaks, CA: Sage

De la Fuente, J., Pichardo, M. C., Justicia, F., y Berbén, A. (2008). Enfoques de aprendizaje, autorregulación y rendimiento en tres Universidades europeas. Psicothema, 20(4), 705-711.

Diener, E., Emmons, R., Larsen, R. J., y Griffin, S. (1985). The Satisfaction With Life Scale. Journal of Personality Assessment, 4, 71-75.

Diseth, Å., Danielsen, A. G., y Samdal, O. (2012). A path analysis of basic need support, selfefficacy, achievement goals, life satisfaction and academic achievement level among secondary school students. Educational Psychology, 32, 335-354.

Domènech Betoret, F., y Gómez Artiga, A. (2011). Relación entre las Necesidades Psicológicas del Estudiante, los Enfoques de Aprendizaje, las Estrategias de Evitación y el Rendimiento. Electronic Journal of Research in Educational Psychology, 9(2), 463-496.

Entwistle, N. J. (1985). A model of the teaching-learning process derived from research on student learning. Paper presented at the international conference of cognitive processes in student learning. Lancaster: University of Lancaster.

Entwistle, N. J. (1987). A model of the teaching-learning process. En Richardson, J. T. E., Eysenck, M. W., y Warren Piper, D. (eds.), Student Learning: Research in Education and Cognitive Psychology. London: S.R.H.E./Open University Press, pp. 13-28.

Gargallo, B. (2006). Estrategias de aprendizaje, rendimiento y otras variables relevantes en estudiantes universitarios. Revista de Psicología General y Aplicada, 59(1-2), 109-130.

Gargallo, B. (2008). Estilos de docencia y evaluación de los profesores universitarios y su influencia sobre los modos de aprender de sus estudiantes. Revista Española de Pedagogía, 241, 425-445.

Gargallo, B. (2009). Los procesos de enseñanza-aprendizaje en la universidad. Revista Educación y Pedagogía, 19(47).

Gillet, N., Vallerand, R. J., y Rosnet, E. (2009). Motivational clusters and performance in a real-life setting. Motivation and Emotion, 33, 49-62. 
González Geraldo, J. L., del Rincón Igea, B., y Delio, A. (2011). Estructura Latente y Consistencia Interna del R-SPQ-2F: Reinterpretando los Enfoques de Aprendizaje en el EEES. Revista de Investigación Educativa, 29(2), 277-293.

Hair, J. F., Anderson, R. E., Tatham, R. L., y Black, W. C. (1998). Multivariate data analysis. New Jersey: Prentice Hall. Traducido al castellano en "Análisis multivariante". Editorial Prentice Hall, 1999.

Harter, S. (1981). A new self-report scale of intrinsic versus extrinsic orientation in the classroom: Motivational and informational components. Developmental Psychology, $17,300-312$.

Hernández, F., García, M. P., y Maquilón, J. (2004). Análisis del cuestionario de proceso de estudio-2 factores de Biggs en estudiantes universitarios españoles. Revista Fuentes, 6, 96-114.

Hernández F., Rodríguez, M., Ruíz, E., y Esquivel, J. (2010). Enfoques de aprendizaje en alumnos universitarios de la titulación de Ciencias de la Actividad Física y del Deporte de España y México". Revista Iberoamericana de Educación, 53/7. Disponible en URL http://www.rieoei.org/3426.htm (Consulta, 23/06/2011)

Hernández Pina, F. (2000). Acceso desde la educación secundaria a la universidad. La calidad del aprendizaje. Problemática y alternativas de mejora. En J. Cajide, M. A. Santos y A. Porto (Ed.), Calidad educativa y empleo en contextos multiculturales. Santiago: Universidad de Santiago. Servicio de Publicaciones.

Hernández Pina, F., Rosário, P., Cuesta Sáez de Tejada, J. D. Martínez Clares, P., y Ruiz Lara, E. (2006). Promoción del aprendizaje estratégico y competencias de aprendizaje en estudiantes de primero de universidad. Revista de Investigación Educativa, 24(2), 615632.

Huebner, E. S., Valois, R. F., Paxton, R. J., y Drane, J. W. (2005). Middle school student's perceptions of quality of life. Journal of Happiness Studies, 6(1), 15-24.

Katz, I., Assor, A., y Kanat-Maymon, Y. (2008). A projective assessment of autonomous motivation in children: Correlational and experimental evidence. Motivation and Emotion, 32, 109-119. doi: 10.1007/s11031-008-9086-0

Kowal, J., y Fortier, M. S. (2000). Testing relationships from the hierarchical model of intrinsic and extrinsic motivation using flow as a motivational consequence. Research Quarterly for Exercise and Sport, 71, 171-181

Kusurkar, R., Croiset, G., Galindo-Garré, F., y Ten Cate, O. (2013). Motivational profiles of medical students: Association with study effort, academic performance and exhaustion. Biomédicalcenter (BMC) Medical Education, $13,87$.

Lent, R. W . (2004). Toward a unifying theoretical and practical perspective on well-being and psychosocial adjustment. Journal of Counseling Psychology, 51, 482-509.

Lent, R. W., Singley, D., Sheu, H., Gainor, K. A., Brenner, B. R., Treistman, D., y Ades, L. (2005). Social cognitive predictors of domain and life satisfaction: Exploring the theoretical precursors of subjective well-being. Journal of Counseling Psychology, 52, 429-442.

Lepper, M. R., Corpus, J. H., y lyengar, S. S. (2005). Intrinsic and extrinsic motivational orientations in the classroom: Age differences and academic correlates. Journal of Educational Psychology, 87, 184-196. 
Lounsbury, J. W., Park, S. H., Sundstrom, E., Williamson, J. M., y Pemberton, A. E. (2004). Personality, career satisfaction, and life satisfaction: Test of a directional model. Journal of Career Assessment, 12(4), 395-406.

Lin, Y. G., McKeachie, W. J., y Kim, Y. C. (2003). College student intrinsic and/or extrinsic motivation and learning. Learning and Individual Differences, 13, 251-258.

Monereo, C. (Coord.) (2000). Estrategias de aprendizaje. Madrid: Visor.

Miquelon, P., y Vallerand, R. J. (2006). Goal Motives, well-being, and physical health: Happiness and self-realization as psychological resources under challenge. Motivation \& Emotion, 30, 259-272.

Miquelon, P., Vallerand, R. J., Grouzet, F. M. E., y Cardinal, G. (2005). Perfectionism, academic motivation, and personal adjustment: an integrative model. Personality and Social Psychology Bulletin, 31, 913-924.

Mpofu, E., y Oakland, T. (2001). Predicting school achievement in African school settings using Bigg's Learning Process Questionnaire. South African Journal of Psychology, 31, 20-28.

Muñoz, E. y Gómez, J. (2005). Enfoques de aprendizaje y rendimiento académico de los estudiantes universitarios. Revista de Investigación Educativa, 23(2), 417-432

Norusis, M. J. (1992). SPSS/PC+ Professional statistics, Version 5.0.Chicago, IL: SPSS.

Núñez, J. L., Martín-Albo, J., y Navarro, J. G. (2005). Validación de la versión española de la Échelle de Motivation en Éducation. Psicothema, 17(2), 344-349.

Ojeda, L., Flores, L. Y., y Navarro, R. L. (2011). Social cognitive predictors of Mexican American college students' academic and life satisfaction. Journal of Counseling Psychology, 58, 61-71.

Pintrich, P. R. (2004). A Conceptual Framework for Assessing Motivation and Self-Regulated Learning in College Students. Educational Psychology Review, 16(4), 385-407.

Pozo, J. I., Monereo, C. y Castelló, M. (2005). El uso estratégico del conocimiento. En: Coll, C., Palacios, J. y Marchesi, A. (Comp.) Desarrollo psicológico y educación. 2. Psicología de la educación escolar. Madrid: Alianza. Psicología y Educación.

Ratelle, C. F., Guay, F., Vallerand, R. J., Larose, S., y Senecal, C. (2007). Autonomous, controlled, and amotivated types of academic motivation: A person-oriented analysis. Journal of Educational Psychology, 99, 734-746.

Recio, M. A., y Cabrero, J. (2005). Enfoques de aprendizaje, rendimiento académico y satisfacción de los alumnos en formación en entornos virtuales. Revista píxel-Bit, Revista de Medios y Educación, 25, 93-115.

Reeve, J., Jang, H., Carrell, D., Barch, J., y Jeon, S. (2004). Enhancing high school students' engagement by increasing their teachers autonomy support. Motivation and Emotion 28, 147-69.

Roeser, R. W., Eccles, J. S., y Sameroff, A. J. (1998). Academic and emotional functioning in early adolescence: Longitudinal relations, patterns, and prediction by experience in middle school. Development and Psychopathology, 10, 321-352.

Rodríguez Ayán, M. (2010). Perfiles motivacionales definidos mediante análisis de conglomerados y su relación con la capacidad percibida y el rendimiento académico. Anales de Psicología, 26(2), 348-358. doi:10.6018/109341 
Rosário, P., Mourão, R., Trigo, J., Núñez, J. C., y González-Pienda, J. (2005). SRL Enhancing Narrtives: Testas (Mis) Adventures. Academic Enchange Quartely, Winter, 9(4), 73-77.

Ryan, R. M., y Connell, J. P. (1989). Perceived locus of causality and internalization: Examining reasons for acting in two domains. Journal of Personality and Social Psychology, 57, 749-761.

Ryan, R. M., y Deci, E. L. (2000). Self-determination theory and the facilitation of intrinsic motivation, social development, and well-being. American Psychologist, 55, 68-78.

Ryan, R. M., y Deci, E. L. (2002). An overview of self-determination theory. En E. L. Deci y R. M. Ryan (Eds.), Handbook of self-determination research (pp. 3-33). Rochester, NY: University of Rochester Press.

Ryan, R. M., Deci, E. L., y Grolnick, W. S. (1995). Autonomy, relatedness, and the self: Their relation to development and psychopathology. En D. Cicchetti y D. J. Cohen (Eds.), Developmental psychopathology: Theory and methods (pp. 618-655). New York: Wiley.

Sheldon, K. M. y Elliot, A. J. (1999). Goal striving, need-satisfaction, and longitudinal wellbeing: The Self-Concordance Model. Journal of Personality and Social Psychology, 76, 482-497.

Suárez Riveriro, J., Fernández Suárez, P., y Anaya Nieto, D. (2005). Un modelo sobre la determinación motivacional del aprendizaje autorregulado. Revista Educación, 338, 295-306.

Vallerand, R. J. (2007). Intrinsic and extrinsic motivation in sport and physical activity. En G. Tenenbaum y R. C. Eklund (Eds.), Handbook of sport Psychology ( $3^{\mathrm{a}}$ ed., pp. 59-83). New York: Wiley.

Vallerand, R. J., Blais, M. R., Briere, N. M., y Pelletier, L. G. (1989). Construction and validation of the motivation toward education scale. Canadian Journal of Behavioural Science Revue Canadienne, 21, 323-349.

Vansteenkiste, M., Soenens, B., Sierens, E., Luyckx, K., y Lens, W. (2009). Motivational profiles from a self-determination perspective: The quality of motivation matters. Journal of Educational Psychology, 101, 671-688.

Wormington, S. V., Corpus, J. H., y Anderson, K.G. (2011). A Person-Centered Investigation of Academic Motivation, Performance, and Engagement in a High School Setting. Learning and Individual Differences, 22(4), 429-438. 


\section{Autores}

Juan Antonio Moreno-Murcia

Doctor en Psicología por la Universidad de Valencia. Actualmente es Profesor Titular de Universidad y ejerce su labor docente e investigadora en la Universidad Miguel Hernández de Elche en el Departamento de Psicología de la Salud.

Sus principales líneas de investigación están relacionadas con la motivación.

Yolanda Silveira Torregrosa

Doctora en Psicología de la Salud por la Universidad de Elche.

Miembro colaborador en el Departamento de Psicología de la Salud de la Universidad Miguel Hernández de Elche.

Su principal línea de investigación está relacionada con la motivación. 